\title{
PERSPECTIVAS ACTUALES PARA EL FOMENTO DEL AULA TRANSCULTURAL EN CHILE
}

\section{CURRENT PERSPECTIVES FOR THE PROMOTION OF THE TRANSCULTURAL CLASSROOM IN CHILE}

\author{
Gloria Toledo Vega \\ Pontificia Universidad Católica de Chile \\ gtoledo@uc.cl \\ Francisco Quilodrán \\ Pontificia Universidad Católica de Chile \\ fgquilodrán@uc.cl \\ Miguel Olivares \\ Universidad de Playa Ancha de Ciencias de la Educación \\ miguel.olivares@alumnos.upla.cl \\ Julio Silva \\ Pontificia Universidad Católica de Valparaíso \\ Juliosilvac1@gmail.com
}

\begin{abstract}
Resumen:
El presente artículo es una revisión selectiva y crítica de literatura sobre el desarrollo de aulas transculturales en contexto escolar. En él, se presenta una visión situada sobre perspectivas y enfoques actuales para las aulas plurilingües en Chile y se ofrece una propuesta concreta, sencilla y clara para la implementación de clases de español como lengua extranjera (ELE) en la escuela pública local. Tras integrar la información sobre la realidad chilena, las perspectivas internacionales actuales, el papel de la escritura en el desarrollo de la competencia en ELE y el currículo educacional chileno, se concluye que es importante contextualizar en el plano local las propuestas académicas (generalmente anglosajonas) y que es imprescindible la enseñanza de ELE para los estudiantes no hispanohablantes, si se quiere potenciar realmente la transculturalidad en los establecimientos educacionales públicos.
\end{abstract}

Palabras clave: ELE, estudiantes inmigrantes, pluricultural, escuela chilena.

\begin{abstract}
:
This article is a selective and critical review of literature on the development of intercultural classrooms in a school context. It presents a situated point of view on current perspectives and approaches for multilingual classrooms in Chile, and offers a concrete, simple and clear proposal for the implementation of classes of Spanish as a foreign language (SFL) in the local public school. After integrating information on the Chilean reality, current international perspectives, the role of writing in the development of SFL competence, and the Chilean educational curriculum, it is concluded that it is important to contextualize academic trends (generally Anglo-Saxon) and that SFL teaching is essential
\end{abstract}


Artículo. Gloria Toledo Vega, Francisco Quilodrán, Miguel Olivares, Julio Silva. "Perspectivas actuales para el fomento del aula transcultural en Chile”.

for non-Spanish-speaking students if transculturality is to be really promoted in public educational establishments.

Keywords: SFL, immigrant students, multicultural, Chilean school.

Recibido: 4 de septiembre de 2020

Aceptado: 9 de noviembre de 2020

\section{Introducción}

Este artículo tiene como objetivo plantear una discusión teórica sobre la enseñanza de segundas lenguas a inmigrantes en contexto escolar, a partir de una revisión bibliográfica extensa y detallada que permite posicionar el caso puntual que se desarrolla. Con este fin se examinó una extensa bibliografía que aborda enfoques vigentes sobre el tema en estudio: la transculturalidad, la multicompetencia y los enfoques de enseñanza de segundas lenguas mediante contenidos.

De acuerdo al informe del Centro de Estudios e Incidencias sobre Acceso a Educación (Roessler, Salas y Ramaciotti 1), entre 2014 y 2019, la matrícula migrante en Chile aumentó en un $616 \%$, concentrándose la mayoría en algunos establecimientos públicos. El informe advierte que esto puede propiciar exclusión, por ser estos establecimientos catalogados como "solo para migrantes". En su reporte Roessler et al. (2) informan de dos puntos críticos: a) potenciales fenómenos de segregación y, b) mermas existentes en la educación formal. Sobre este último punto, Roessler et al. (2) proponen establecer una respuesta institucional para la enseñanza del idioma local a estudiantes no hispanohablantes. Para responder al último punto, este artículo aborda tres objetivos: (1) poner el foco de la inmersión escolar hablante no nativa de español en la enseñanzaaprendizaje de ELE; (2) analizar con atención a nuestro contexto particular la aplicación de enfoques y perspectivas para la enseñanza escolar a inmigrantes; y (3) entregar una propuesta clara, realista y organizada para la enseñanza de ELE en establecimientos públicos con alta concentración de estudiantes no hispanohablantes.

La revisión bibliográfica que aquí se presenta está mediada por la experiencia docente e investigativa en ELE de sus autores y por el trabajo de estos en el proyecto Fondecyt Regular $n^{\circ} 1190254$. Dicho trabajo es un estudio de caso sobre el desarrollo de interlengua en estudiantes secundarios haitianos y se traduce en encuestas, entrevistas y grupos focales 
a profesores, directores, sostenedores y autoridades regionales de escuelas públicas y subvencionadas, llevadas a cabo en el transcurso de dos años. La investigación se concentra en un colegio con alta concentración de estudiantes haitianos, donde se efectuó un análisis de errores de las producciones escritas de todos los estudiantes de I a IV medio. Las profesoras de este colegio y otros profesores de Lenguaje externos revisaron mediante una pauta las muestras escritas, de forma tal que pudimos hacer un meta análisis de cómo eran evaluados los estudiantes hispanohablantes y haitianos. Este proyecto aún necesita el análisis de otras muestras diferidas en el tiempo para ver si existe desarrollo de la competencia escrita entre los estudiantes no hispanohablantes. Por ahora, nos basamos en los resultados del primer análisis de errores, en las entrevistas realizadas durante dos años y en una extensiva revisión bibliográfica.

\section{Desarrollo y discusión}

\subsection{Problemas en aulas plurilingües y revisión crítica de enfoques para la enseñanza de $\mathbf{L} 2$}

\subsubsection{Transculturalidad e interlengua}

Una representación usual en la comunidad escolar es aquella que tiende a pensar que los hablantes no nativos de una lengua tienen problemas con las asignaturas de la escuela porque sufren algún tipo de déficit cognitivo. Este parecer se pudo comprobar en las entrevistas y grupos focales que sostuvimos con profesores y autoridades de escuelas, y aparece registrado en Collier y Hoover (39-45), Ochoa, Rivera y Powell (161-167), Rhodes, Ochoa y Ortiz (57) y Pearson (274-277), entre otros. Sobre lo dicho, se debe considerar que difícilmente un estudiante que desconoce la lengua meta podría acceder a los contenidos disciplinares que debe revisar en la escuela, si esos contenidos son entregados en una lengua que no conoce. No se trata de un problema cognitivo, sino de un problema de acceso a la enseñanza-aprendizaje de L2. Esta perspectiva deficitaria sobre los hablantes no nativos $(\mathrm{HNN})$ no contempla la posible existencia de tradiciones retóricas diferentes en su lengua materna (L1). La Retórica Contrastiva [RC] (Kaplan) tuvo el mérito de reconocer la 
Artículo. Gloria Toledo Vega, Francisco Quilodrán, Miguel Olivares, Julio Silva. "Perspectivas actuales para el fomento del aula transcultural en Chile”.

lengua materna de un hablante y, con ello, sus tradiciones retóricas; sin embargo, no le prestó mayor atención a las influencias de grupos, lenguas y tipologías textuales dominantes en un contexto de enseñanza-aprendizaje de segunda lengua [L2], temas que más recientemente han abordado Canagarajah (Translingual), Kubota (Cross-cultural) y Kubota y Lehner (Toward; Response). Frente a esta insuficiencia, se propuso la evolución del concepto de RC al de Retórica Intercultural [RI], (Connor, Intercultural); (Connor, Nagelhout y Rozycki, Contrastive), cuya orientación va más allá de la comparación de textos escritos en L1 y L2, e incluye un foco multimodal en la interacción de escritores y hablantes. La RI entiende los textos escritos en L2 como el producto de una interlengua conformada por elementos de la L1, la L2 y otras posibles lenguas adicionales del aprendiente. La noción de interlengua (Selinker), en tanto, ha servido a investigadores que han estudiado este constructo inestable e idiosincrásico en base a metodologías como el análisis de errores, con lo cual han podido dar cuenta del desarrollo hacia la lengua meta. En una perspectiva post-estructuralista, autores como Canagarajah (The Routledge 3) o Blackedge y Creese (The Routledge 31-32) proponen cambiar el foco de las prácticas interlingüísticas a otras translingüísticas, que tomen en cuenta el concepto de movilidad. Según estos autores, la interlengua atiende a grupos estáticos y compara invariablemente las producciones en L2 con la lengua meta. Las prácticas translingüísticas, por el contrario, atenderían mejor a la naturaleza activa de la negociación y se alinearían con los contextos globales actuales en que los comunicadores comparten distintas lenguas y culturas.

Desde nuestro punto de vista, la lengua meta es un componente más del marco tripartito en el cual se basa la metodología de análisis de errores (L1 - IL - L2). Considerando lo anterior, la tradición investigativa no parece ser el problema, sino cómo esta se articula con las prácticas pedagógicas en contextos concretos y reales. Ahora bien, tanto desde la visión de la IL como de la transculturalidad, se entiende que hay procesos de adquisición de una segunda lengua que son absolutamente normales; interferencia, periodo silente, cambio de código, pérdida del lenguaje y producciones idiosincráticas (RoseberryMcKibbin 193). Por lo mismo, desde ambos puntos de vista queda claro que no existen HNN defectuosos, como tampoco hablantes nativos (HN) ideales. En esta revisión, centrada en la enseñanza-aprendizaje de ELE, se entenderá que la lengua meta es la lengua por aprender; que el hablante no nativo es el aprendiente de la lengua meta y que el 
hablante nativo es quien habla la lengua meta, pero de ninguna forma el modelo a seguir o el hablante ideal, como veremos más adelante.

\subsubsection{El aprendiente multi-competente}

De acuerdo con Cots et al. (24), existen tres modelos que se adecuan a la situación de plurilingüismo escolar: el modelo dinámico de multilingüismo (Herdina y Jessner); el modelo de multi-alfabetización (Cummins, De la alfabetización) y el modelo de multicompetencia (Cook, Premises), sobre el que nos detendremos más. El modelo de multilingüismo sostiene que la competencia de los sujetos multilingües consta de subsistemas lingüísticos dinámicos, que interactúan entre sí (L1, L2, L3...) y que están sujetos a variación. En esta perspectiva, es importante considerar que aunque ser multilingüe es una ventaja desde varios puntos de vista, también puede retrasar los procesos de desarrollo de las habilidades lingüísticas. Este atraso no debe ser visto como un déficit, sino como un proceso natural.

El modelo de multi-alfabetización consiste en una pedagogía que busca cristalizar en la enseñanza la identidad en varias lenguas. Al respecto, Cots et al. (28) ponen como buen ejemplo el de Canadá, país que es en sí mismo una sociedad bilingüe. En el caso de Chile, que históricamente ha relegado las lenguas indígenas y que muestra falencias en la enseñanza-aprendizaje del inglés (Education First) y de otras lenguas adicionales, la situación es la de una sociedad monolingüe.

El modelo de multi-competencia (Cook, Premises) valora los beneficios lingüísticos y cognitivos de poseer el conocimiento de más de una lengua, e intenta recuperar el uso de la lengua materna en la enseñanza de L2. Cook (Premises), Lorente y Canagarajah (The Routledge), entre otros autores, critican las prácticas monolingües en la sociedad o en comunidades como la escuela. En la sociedad chilena o latinoamericana, sin embargo, el monolingüismo es una realidad, debida, en gran parte, a la escasez de recursos. En Chile, como en otros países de la región, el que no domina o no conoce una L2 muy probablemente será alguien que no ha podido acceder a educación de calidad. Alguien que por falta de recursos económicos no ha podido viajar fuera de su país y quien con dificultad 
podrá acceder, dentro de su propia cultura, a esferas socioculturales distintas a las de la comunidad socioeconómicamente vulnerable en la que vive.

En cuanto al papel del aprendiente en la perspectiva de Cook, este se escinde totalmente de la categoría de usuario de una L2, bajo el entendimiento de que el concepto de aprendiente es el de una persona con capacidades siempre incompletas en relación con el hablante nativo (Multi-competence 447; Premises 4). Para reconciliar el estatus de aprendiente con la perspectiva de la multi-competencia, Scott (448) propone el concepto de aprendiente multicompetente. Conforme a esta idea, a la cual adherimos en este trabajo, un individuo que conoce dos o más lenguas puede ser usuario y aprendiente de ellas. Dado que el mismo Cook propone que los usuarios de L2 sean su propio modelo en lugar de los hablantes nativos (Premises 35), estimamos que su visión respecto al aprendiente no tiene por qué ceñirse a un parámetro monoglósico. En nuestro estudio, cuando quisimos comparar a los estudiantes haitianos respecto a los hablantes nativos de español (chilenos, ecuatorianos, colombianos y venezolanos en un mismo curso), comprobamos que las producciones escritas de los hispanohablantes distaban mucho de ser un modelo a seguir. Poner dicho parámetro no solo habría sido injusto, en términos de la pobreza de la comparación, sino que además poco propicio desde el punto de vista didáctico y pedagógico.

Los tres modelos que hemos señalado (multi-alfabetización, multilingüismo y multicompetencia) son complementarios, trascienden la competencia comunicativa (Canale; Bachman) y se enfocan en la competencia multicultural. Todos los modelos se ajustan a una visión post-estructuralista y sociocultural, y a un enfoque pedagógico socioconstructivista que considera que una lengua se actualiza en prácticas socio-comunicativas concretas. De los tres modelos, sin embargo, solo el de multi-alfabetización se centra en prácticas didácticas y pedagógicas concretas.

\subsection{Perspectivas pedagógicas vigentes para el aprendizaje-enseñanza de una L2}

Entre los enfoques pedagógicos tendientes a crear un ambiente rico en discurso instruccional, Lyster (56-58) alude a perspectivas holísticas como Process Writing, el Lenguaje a Través del Currículum o la Enseñanza Mediante Contenidos. En esta revisión 
destacamos la última perspectiva, por estar más directamente enfocada hacia el aprendizaje de una lengua adicional.

El Aprendizaje Integrado de Contenidos y Lenguas Extranjeras (AICLE); Enseñanza a Través de Contenidos; o Programa de Inmersión Dual, es un enfoque pedagógico que atiende al fomento de clases trans linguales. Este tipo de enseñanza vincula el aprendizaje de una L2 con los contenidos de una asignatura, organizados temáticamente alrededor de tres conceptos claves: géneros hablados y escritos, registros específicos y multi-modalidad. Corson (323) advierte que este enfoque apunta al desarrollo del lenguaje a través de su utilización con un propósito claro, en que interactúan la enseñanza-aprendizaje de la lengua y el ámbito académico. Es muy probable que el mejor ejemplo de aplicación de este tipo de aprendizaje se encuentre en las escuelas bilingües canadienses, que cuentan con una sociedad nacional bicultural y bilingüe, y con un amplio despliegue de recursos destinados a la educación. En un contexto óptimo como este, la enseñanza mediante contenidos puede ser bilingüe, como en los colegios privados bilingües o internacionales en Chile.

Dentro de los sub-enfoques que se pueden encontrar en AICLE, destacamos el enfoque contrabalanceado (Counter-balanced approach) que presenta Lyster (125). Sobre esto, el autor destaca cómo las opciones instruccionales más efectivas, tanto para HN como para $\mathrm{HNN}$, son aquellas que tienen que ver con el fomento de la interacción, el uso del lenguaje y la corrección explícita. No obstante, su aplicación exitosa está sujeta a condiciones que difícilmente pueden cumplir los establecimientos educacionales públicos en Chile, según veremos a continuación.

La implementación del esquema de enseñanza-aprendizaje mediante contenidos cubre las necesidades de HNs y de HNNs, para lo cual requiere de especialistas en este enfoque. Para que funcione un enfoque de este tipo es necesario que el profesor sepa: cómo y cuándo modificar el input que entrega a los estudiantes; cómo y cuándo entregar respuestas semánticamente contingentes; generar modificaciones conversacionales (confirmaciones de comprensión y clarificaciones) y tener un conocimiento funcional de la gramática española, además de aptitudes relacionadas con la valoración y el tratamiento de la diversidad en el aula. Más importante aún, para que este enfoque pueda funcionar verdaderamente, es necesario que los hablantes no nativos de la lengua meta tengan una instrucción formal mínima sobre esta y que esta instrucción garantice un nivel lo más parejo posible entre los 
HNN de un curso. Ningún enfoque o metodología logrará que en una misma clase hispanos y no hispanohablantes aprendan contenidos disciplinares y participen activamente, si los últimos individuos no tienen un conocimiento de la lengua que posibilite la interacción. Idealmente, los $\mathrm{HN}$ podrían tener conocimientos de la lengua materna de los haitianos (creole), pero desde un punto de vista realista esto es complejo si históricamente no se ha atendido bien a la enseñanza de lenguas adicionales. Respecto a la nivelación de la L2, nuestra investigación muestra que no existe un conocimiento uniforme de español entre los haitianos de un mismo curso; es más, pudimos observar el caso de una estudiante no alfabetizada que cursaba segundo medio. Estas situaciones propician que de la escuela egresen estudiantes con una competencia muy desigual en español, que saldrán al mundo laboral con mínimas herramientas para asegurar su bienestar socioeconómico. Considérese además la gran cantidad de alumnos por clases y lo complejo que es nivelar una clase con más de 40 individuos, atendiendo a niveles de lengua entre HNN y al rendimiento escolar entre HNN y HN. Según la hipótesis del umbral de Cummins (The influence; Language), los aspectos del bilingüismo que influencian positivamente el desarrollo metalingüístico y cognitivo en un individuo funcionan poco si este no ha alcanzado un nivel umbral en la lengua meta.

Por último, tómese en cuenta la complejidad morfosintáctica del español. Lyster (29) registra que las aplicaciones del enfoque mediante contenidos han sido más exitosas en relación con el inglés que con el francés, lengua esta última que comparte rasgos morfosintácticos de dificultad muy similares al español. Estas complejidades podría afrontarlas un profesor de Lenguaje, pero difícilmente un profesor de otra asignatura, lo cual limita el enfoque inevitablemente en cuanto a contenidos disciplinares.

Llegados aquí, nos interesa hacer hincapié en que es fundamental tomar en cuenta la enseñanza-aprendizaje de ELE como especialidad complementaria en la educación. No es posible soslayar la enseñanza de la lengua meta en un contexto como la escuela. La competencia en una lengua posibilita la formación del usuario competente que propugna Cook. En efecto, el lenguaje es una herramienta que permite el acceso a la educación superior, a la posibilidad de conseguir o negociar mejores trabajos, a ser agente en la participación comunitaria y en la (re)formulación de la identidad propia. Lo anterior, incide en la mejora de la autoestima y del potencial individual (Pavlenko; Cots et al. 39; 
Canagarajah, Lingua 936; Urciuoli y LaDousa 176; Zhu, Interculturality 121). Sobre esta visión del lenguaje, el Marco Común Europeo de Referencia (Consejo de Europa) sostiene que participar en una relación intercultural implica que "una persona, en cuanto agente social, domina varias lenguas y posee experiencias de varias culturas" (Consejo de Europa 167). A diferencia de un individuo bicultural o multicultural, resultado del proceso natural de habitar en distintos contextos socioculturales, el sujeto intercultural ha experimentado un proceso de educación al que Byram (110) llamó “tercera socialización”. Este proceso, le permite al individuo tener consciencia de su lugar en la comunidad, relacionar culturas distintas y mediar entre sus miembros.

\subsubsection{Aplicaciones didácticas para la enseñanza-aprendizaje en un aula pluricultural}

El foco en el estudiante y en el aprendizaje impone una actividad más desafiante para el docente, dado que el profesor debe detectar y aprovechar las oportunidades de aprendizaje, al tiempo que la clase avanza y se desarrolla, para involucrar a los aprendientes en negociaciones, clarificaciones, confirmaciones o reformulaciones (Shepherd 166). Lo anterior necesita flexibilidad curricular y atender a las habilidades más que a los contenidos. Esto descomprimirá las programaciones y daría tiempo a implementar proyectos que involucren a los estudiantes en su desarrollo. Los proyectos, a su vez, mediarían en la aplicación de los conocimientos y motivarían a los HNN a ser usuarios y aprendientes multi-competentes, sin descuidar el progreso de los hablantes monolingües. Un ejemplo de trabajo con proyectos lo entrega Dakin (20), quien explora los beneficios que conlleva la redacción de historias personales de estudiantes inmigrantes. Actividades como estas no solo involucran a los aprendientes en un plano académico y emocional, sino también les permite a los profesores tener un mejor conocimiento de sus estudiantes.

Kaneva relata experiencias exitosas en la integración del alumnado inmigrante en escuelas inglesas que han procurado la implementación de profesores de inglés como lengua adicional y personal bilingüe. Ahora bien, este apoyo de profesores especializados y personal bilingüe no es suficiente por sí solo. Las escuelas aventajadas en la inmersión del alumnado inmigrante han entendido que las clases de apoyo lingüístico a los HNN son una necesidad. Si bien Kaneva (62) aclara que no hay una estrategia directa que funcione en 
todos los contextos, existe acuerdo en que las clases de apoyo ayudan a los estudiantes en su desarrollo como aprendices de lengua y de contenidos. Al respecto, Collier y Thomas (336) sugieren que para el éxito académico de los estudiantes es importante que estos aprendan la L2 mientras progresan en su currículum escolar. Esto deja la responsabilidad del aprendizaje de la L2 a la escuela porque, sin duda, este es el mejor lugar para que converjan el aprendizaje de la lengua y de los contenidos disciplinares. Para lograr esta empresa, es necesario que los esfuerzos a largo plazo tiendan al tratamiento integrado de las lenguas en el currículo; a la coordinación entre el profesorado, y a la existencia de un proyecto lingüístico central. Cots et al. (98) recogen estos tres puntos entre el profesorado español de escuelas con estudiantes inmigrantes $\mathrm{y}$, atendiendo a las numerosas entrevistas y grupos focales que hemos llevado a cabo como grupo de estudio, podemos atribuir las mismas necesidades a los profesores chilenos.

\subsection{La escritura en una lengua adicional: una vía de desarrollo del lenguaje en un aula pluricultural}

La escritura es importante, porque se asocia con el pensamiento crítico y con el uso de tiempo y energía en actividades cognitivas tales como planear y buscar información (Grabowski 80-81). El acto de escribir hace visible el pensamiento, obliga a reflexionar sobre un tema y permite que el estudiante modifique, precise y aclare las ideas y los conocimientos que tenía previamente (National Writing Project y Nagin 43). En la escritura, a diferencia de la comunicación oral, el escritor carece de los apoyos contextuales propios del lenguaje hablado, por lo que debe tomar decisiones sobre múltiples variables para asegurar que su lector lo entienda (Bereiter y Scardamalia 102). Esta habilidad ayuda a desarrollar la consciencia sintáctica y, junto a esta, la comprensión lectora (Bizama, Arancibia y Saez; Mata, Gallego y Mieres) y a expandir la diversidad léxica de los aprendices. No obstante todo lo señalado, los datos de la prueba PISA 2018 en nuestro país muestran que un $31,7 \%$ de los estudiantes de $7^{\circ}$ básico no poseen habilidades adecuadas de comprensión literal e inferencial, y solo un 2,6\% de los estudiantes del territorio nacional presenta un rendimiento dentro de lo esperado en esta competencia (Agencia de la calidad de la Educación). 
Escribir en la lengua nativa implica ejercitar una versión especializada de un lenguaje. Esta especialización plantea diferencias tanto en la forma como en el uso, y su meta final es ser capaz de participar de forma completa en aspectos sociales más allá de la escuela; entre otros, el acceso a la educación superior. Esta actividad, en el caso de estudiantes HNN, plantea la dificultad de no contar con los recursos lingüísticos que los $\mathrm{HN}$ ya poseen (Cushing 4), para movilizar conocimientos gramaticales, textuales, funcionales y sociolingüísticos (Bachman y Palmer, Language 70; Douglas 35).

En contextos plurilingües, las actividades de escritura son una forma accesible para profesores y estudiantes para revisar contenidos disciplinares y de lenguaje. Los profesores de Lenguaje -a quienes se les ha asignado la compleja tarea de guiar a los HNN en la escuela chilena- pueden valerse de la escritura y de la retroalimentación de esta para evaluar, guiar y potenciar el desarrollo en español de sus estudiantes no hispanohablantes. Para esto, se debe conceder mayor importancia a la escritura y propiciar tareas de escritura que respondan a criterios bien establecidos: qué habilidad se quiere explotar, con qué propósito se escribe, qué secuencia textual se va a desarrollar y para qué contexto específico. También es importante el input de estímulo que se entregará a los estudiantes y la forma en que este será modelado. Smith, Hull y Land, por ejemplo, descubrieron en su investigación que los estudiantes se desempeñan mejor reaccionando a varios extractos breves, en lugar de solo uno extenso. El estudio de Lewkowicz, por otra parte, muestra que cuando a los estudiantes se les entrega un texto como respaldo para la redacción hay un efecto limitante, pues tienden a copiar y/o parafrasear más.

\subsubsection{Evaluación de la escritura atendiendo a la diversidad en el aula}

Una tarea de escritura, por más bien pensada que esté, no servirá de mucho si no es retroalimentada por los profesores. Esto nos lleva al problema de cómo evaluar las producciones de los HNN. Para los profesores de escuela en clases pluriculturales y lingüísticamente diversas es un dilema transversal el cómo evaluar a los HNN (Slomp 88). Las evaluaciones estandarizadas, que siguen nociones de validez psicométrica, representan otra complejidad en este escenario, pues no toman en cuenta los contextos plurilingües. En Chile los haitianos, o cualquier estudiante HNN, deben rendir el SIMCE. El rendimiento de 
Artículo. Gloria Toledo Vega, Francisco Quilodrán, Miguel Olivares, Julio Silva. "Perspectivas actuales para el fomento del aula transcultural en Chile".

esta prueba, con escasos conocimientos de la lengua meta y aún menos conocimientos disciplinares, baja los promedios del establecimiento educacional, lo que hace que este se vea afectado en la asignación de recursos económicos y en su reputación. Cuando se somete a los estudiantes HNN a dispositivos estandarizados, se asume que estos son iguales a los estudiantes que han servido de parámetro; en otras palabras, se asume su aculturación (Rhodes, Ochoa y Ortiz 127). Una justa interpretación de las habilidades cognitivas debiera ser en la lengua del aprendiente, pero el acceso al creole en Chile, entre los evaluadores, es complejo. Una prueba en francés (L2 de los haitianos) tampoco garantizaría un mejor desenvolvimiento de los haitianos, pues su competencia en francés depende mucho de la situación socioeconómica previa a su llegada a Chile. En otras palabras, aquellos que gozaban de mejor situación económica son quienes recibieron una mejor formación en francés, con lo cual, sería injusto aplicar una prueba en esta lengua. Quizás debiéramos cuestionarnos más y mejor si acaso las pruebas estandarizadas realmente sirven para algo positivo; si acaso cumplen con alguna meta pedagógica fuera de "ranquear" los colegios. Para Huot (557) una evaluación justa solo puede lograrse de forma local, conociendo bien a las personas, sus prácticas y propósitos involucrados en la escritura. Ahora bien, esto pone a los profesores en una tensión entre atender a las características de cada uno, sin enfocarse en los aspectos deficitarios, y sin caer en una visión paternalista que los lleve a reducir contenidos o a mermar las prácticas académicas más desafiantes (Mehmedbegović 72-73; Hawkings y Cannon 529). Los trabajos de Cummins, (Language; Negotiating) apuntan a que es necesario entregar a los aprendientes experiencias que no estén plagadas de modelos estereotípicos deficitarios y centrarse en lo que los aprendientes sí tienen.

En cuanto a la calificación, (Hayes 14 - 16) y nuestras propias observaciones a partir de grupos focales y análisis de evaluaciones, indica que los evaluadores inexpertos tienden a revisar más errores locales (a nivel de oración), y no errores globales (relacionados con el contenido y la organización textual), calificando conforme a eso. Es esperable que el decreto 67, que favorece la evaluación formativa en la escuela, se ajuste mejor a las aulas plurilingües, siempre y cuando exista retroalimentación adecuada.

Bajo la perspectiva translingüística, se comprende que la comunicación efectiva no consiste en la aplicación de formas fijas de lenguaje y normas pragmáticas convencionalizadas (Cogo y Dewey 169); sin embargo, existe evidencia de que los 
aprendientes prefieren aprender la variedad estándar de una L2 (Maley 194). Los académicos, por su parte, proponen que los profesores consideren las desviaciones de la norma estándar como el uso creativo de los recursos lingüísticos -y no como errores-, sin embargo, esta mirada requiere un compromiso de tiempo y esfuerzo para que, en primer lugar, los profesores puedan distinguir un error de una innovación creativa (Pearson 118). Esto conduciría a repensar la evaluación de las tareas de escritura; los propósitos que se persiguen con estas y si acaso se aceptarán como válidas las mezclas de códigos, de idiomas o de registros (Canagarajah, Translingual 614). Nuestra experiencia como profesores de ELE nos dice que los estudiantes sí valoran la corrección en la clase, lo que no descarta que también valoren las mezclas de códigos lingüísticos en contextos más familiares. Sobre el acceso a los distintos géneros y registros, Cummins (Bilingualism) distingue dos tipos de uso del lenguaje: las habilidades básicas de comunicación interpersonal, cuya adquisición y la competencia académico-cognitiva, siendo esta última crítica para el progreso académico de los niños. La enseñanza debe brindar acceso a ambas, con nociones claras para el uso adecuado de una y otra.

\subsection{La habilidad del lenguaje y la escritura en las bases curriculares chilenas}

Entre los criterios para la creación de las Bases Curriculares está “aprender a expresarse con claridad y eficacia en forma escrita y oral para comunicarse con otros, llegar a acuerdos, persuadir y desarrollar su propio pensamiento" (MINEDUC 19). Dicho criterio está relacionado con el Objetivo General de "Expresarse [...] en forma clara y eficaz, de modo oral y escrito y tomar conciencia del poder del lenguaje para construir significados e interactuar con otros" (MINEDUC 14). En el plano específico de la asignatura de Lengua y Literatura, el currículum indica que el lenguaje desempeña un papel fundamental en la comunicación, la vida social y el pensamiento, así como en la construcción y transmisión de las culturas (Prior 54-66), el establecimiento y mantención de las relaciones e instituciones humanas, y la formación de identidades individuales y colectivas. A partir de las bases curriculares de la asignatura de Lengua y Literatura, se espera que los estudiantes utilicen la escritura para explorar su creatividad; para elaborar, clarificar y compartir sus ideas y conocimientos; para comunicarse en la vida cotidiana; para desarrollarse en los 
ámbitos personal y académico, y para difundir sus ideas o las de un grupo en contextos públicos de comunicación. Además, se espera que los estudiantes demuestren preocupación por el lector al aplicar correctamente las convenciones lingüísticas, al seleccionar el vocabulario para transmitir los mensajes con precisión y claridad, y al incluir la información necesaria para la comprensión del texto. Atendiendo a todo esto, parece claro que las bases curriculares consideran la escritura como una herramienta primordial al momento de comunicarse; sin embargo, en nuestro estudio se pudo comprobar que los estudiantes (HNN y HN) poseen pocos o nulos conocimientos acerca de estrategias de redacción que los ayuden a escribir de manera clara y/o coherente, y que sus tareas de escritura son muy escasas.

\subsection{Propuesta para la enseñanza de español como lengua adicional en la escuela chilena}

A continuación, presentamos una propuesta para la enseñanza de ELE, adicional a las asignaturas escolares en la escuela chilena. Se trata de un programa contemplado para desarrollarse durante un periodo de 4 meses, idealmente a partir del comienzo oficial de clases. De forma óptima, las clases serían dos días a la semana, durante 14 semanas, y cada clase sería de 2 horas cronológicas. Cada unidad corresponde a cinco clases (10 horas). Las clases se articulan en torno a los contenidos y objetivos curriculares oficiales del MINEDUC y a los objetivos por nivel de ELE. Cada clase contempla el trabajo con las cuatro habilidades lingüísticas, con un énfasis especial en el desarrollo de habilidades de lectoescritura, donde se les pide a los estudiantes que reconozcan, analicen y creen diversos tipos de secuencias textuales (explicaciones, comentarios, justificaciones y argumentaciones, principalmente). Como propósito central, se estimula el pensamiento crítico de los estudiantes en un contexto intercultural que propicia su mejor integración y rendimiento académico. En la Tabla 1, se puede ver la programación para el nivel A2 (básico, según MCER), donde se articulan, resumidos, los objetivos de aprendizaje para la asignatura de Lenguaje y Comunicación según el currículo nacional, y los objetivos funcionales para el nivel A2 de ELE, según el MCER. 
Artículo. Gloria Toledo Vega, Francisco Quilodrán, Miguel Olivares, Julio Silva. "Perspectivas actuales para el fomento del aula transcultural en Chile".

qué están diciendo según la situación comunicativa representada.

d. PROFESOR: Muestra diferentes frases y diálogos que corresponden a las situaciones comunicativas anteriores.

e. ESTUDIANTES: En clase abierta, deben señalar qué frase se conecta con la ilustración.

f. PROFESOR: Hacer una puesta en común destacando el uso del imperativo en diversas situaciones comunicativas.

g. PROFESOR: Presentar la unidad didáctica: aprendizajes esperados, contenidos que se verán y evaluación.

\begin{tabular}{|l|l|l|}
\hline Desarrollo & Tiempo: 90 minutos & $\begin{array}{l}\text { Técnica: Lectura en } \\
\text { voz alta }\end{array}$ \\
\hline
\end{tabular}

Actividades de activación / prelectura (10 minutos):

PROFESOR: Preguntas a clase abierta: ¿Qué opinan ustedes sobre el mundo del trabajo en la actualidad? ¿Qué opinan de los jefes? ¿Cómo los describirían?

ESTUDIANTES: Responden las preguntas voluntariamente o elegidos de manera aleatoria.

PROFESOR: Presentación del tema del texto y de las actividades a realizar.

ESTUDIANTE: Hacer una revisión rápida y subrayar en el texto el vocabulario que no entiendan.

ESTUDIANTE: Usando el diccionario o celular (se recomienda rae.es), buscar el significado de las palabras subrayadas y escribirlo con letra imprenta y clara en un post it que luego los estudiantes pegarán (en orden alfabético) en la pizarra, para formar un banco de palabras.

\section{Lectura en voz alta del texto por parte de estudiantes voluntarios o elegidos (10 minutos)}

ESTUDIANTES: Leen texto: “Cómo impresionar a tu jefe” https://mi-felicidad.com/trabajo/comoimpresionar-jefe.html

\section{Post lectura (40 minutos)}

En grupos de 3 estudiantes, se deberán realizar las siguientes actividades:

ESTUDIANTES: Identificar tipo de texto (expositivo / argumentativo), intención del autor y contexto de producción.

PROFESOR: Preguntar a los alumnos si están de acuerdo o no con lo que se plantea en el texto y que fundamenten su respuesta.

ESTUDIANTES: Marcar en el texto las partes que para ellos significan recomendaciones o instrucciones, poniendo énfasis en los verbos.

ESTUDIANTES: Desde su “intuición”, clasificar los verbos en regulares e irregulares.

ESTUDIANTES: Responden la siguiente pregunta: ¿A qué forma gramatical se parece esta estructura? La respuesta esperada es que relacionen la forma del imperativo con el presente de indicativo.

PROFESOR: Revisar la estructura del imperativo en clase, haciendo el paralelo con la forma de la tercera persona del presente de indicativo. El enfoque de uso en este contexto debe estar en la función de recomendar o dar instrucciones. 


\section{Actividad (30 minutos)}

PROFESOR: En la pizarra, poner una lámina grande con la siguiente frase: Propósitos para el próximo año. ESTUDIANTES: En parejas, cada grupo va a elegir desde una caja una fotografía de un personaje famoso o de actualidad, a quién deberán identificar / reconocer y crearle un "propósito" para el próximo año.

ESTUDIANTES: Redactar 10 consejos o recomendaciones para que este personaje "logre" el propósito creado.

ESTUDIANTES: Cada pareja de estudiantes deberá representar la situación a través de un diálogo simulado, en el que un estudiante es el personaje "famoso" y otro su "amigo/a" que le hace las recomendaciones. Ambos deben reaccionar y argumentar por qué propone / elige / rechaza el consejo o recomendación, para finalmente llegar a un acuerdo.

PROFESOR: Evalúa la actividad según una rúbrica con los indicadores de evaluación correspondientes.

\begin{tabular}{|l|l|l|}
\hline Cierre & Tiempo: 10 minutos & $\begin{array}{l}\text { Técnica: Ticket de } \\
\text { salida }\end{array}$ \\
\hline
\end{tabular}

Para cerrar la sesión, cada estudiante deberá completar el "ticket de salida", respondiendo brevemente las siguientes preguntas: ¿Qué aprendí hoy?; ¿Qué actividad me gustó más?; ¿Qué fue lo más difícil?

\begin{tabular}{|c|c|}
\hline Indicadores de evidencia del aprendizaje & $\begin{array}{l}\text { Técnicas e instrumentos de } \\
\text { evaluación }\end{array}$ \\
\hline $\begin{array}{l}\text { El/la estudiante logra identificar el tipo de texto. } \\
\text { El/la estudiante logra identificar la intención del autor. } \\
\text { El/la estudiante logra identificar el contexto de producción del texto. } \\
\text { El/ la estudiante logra expresar una opinión fundamentada. } \\
\text { El/ la estudiante logra crear un propósito relacionado con el } \\
\text { personaje elegido. } \\
\text { El/ la estudiante utiliza adecuadamente las formas del imperativo } \\
\text { para redactar recomendaciones o dar instrucciones. } \\
\text { El/ la estudiante logra establecer un diálogo coherente con su } \\
\text { interlocutor, de acuerdo con las instrucciones de la actividad. } \\
\text { El/ la estudiante logra utilizar de manera adecuada las formas del } \\
\text { imperativo en el diálogo oral. } \\
\text { El/ la estudiante reacciona adecuadamente a los estímulos orales en } \\
\text { relación con las recomendaciones o instrucciones dadas / recibidas } \\
\text { en el diálogo. }\end{array}$ & $\begin{array}{l}\text { Rúbrica para evaluar decálogo. } \\
\text { Rúbrica para evaluar diálogo } \\
\text { simulado. } \\
\text { Pauta de cotejo para evaluar ticket } \\
\text { de salida. }\end{array}$ \\
\hline
\end{tabular}

Tabla 2: Programación clase 1 de la unidad 
Artículo. Gloria Toledo Vega, Francisco Quilodrán, Miguel Olivares, Julio Silva. "Perspectivas actuales para el fomento del aula transcultural en Chile".

\section{Conclusiones}

El estudio en que se basa este artículo comprende entrevistas, grupos focales, encuestas, análisis de errores en la escritura de haitianos, meta-análisis de las evaluaciones de profesores de Lenguaje a sus estudiantes y una basta revisión bibliográfica. En estas páginas, resumimos algunos datos sobre los aciertos y desafíos que muestran distintas perspectivas y enfoques para la implementación de aulas plurilingües en el mundo y en nuestro país, y la forma en que la escritura contribuye a este propósito. Como contribución propia, destacamos el papel de la escritura y de la evaluación de esta habilidad como una manera efectiva de desarrollar la competencia en ELE. Además, entregamos una propuesta de programación de un curso de ELE para la inmersión social y académica de los adolescentes inmigrantes no hispanohablantes en la escuela de nuestro país.

A partir de todo lo que hemos expuesto, podemos extraer dos conclusiones importantes: en primer lugar, es necesario prestar atención al contexto local en que se desarrollará cualquier perspectiva o enfoque valorado internacionalmente para la implementación de aulas pluriculturales. En segundo lugar, es imprescindible la enseñanza de ELE en cursos plurilingües que aspiran a la transculturalidad. Dichos cursos deben existir dentro de la escuela chilena y de forma adicional a cualquier otra asignatura que deban cursar los estudiantes no hispanohablantes.

El proyecto en el que se enmarca este artículo tiene el valor de considerar la realidad de nuestro país, los recursos que dispone el Estado chileno para la educación y los agentes que participan en ella: estudiantes hispanohablantes (chilenos y extranjeros), estudiantes haitianos, profesores, directores de escuela, sostenedores y autoridades educativas. Aún queda por analizar el desarrollo de interlengua de los estudiantes haitianos, para comprobar su grado de aprendizaje de español, su desarrollo académico en la escuela, su grado de inmersión sociocultural y su proyección como futuros estudiantes de educación superior o trabajadores. Desgraciadamente, hasta el momento no hay señales optimistas respecto a las proyecciones de esta comunidad de estudiantes: aún no se instala la idea de que es absolutamente necesario ofrecerles formación en español como lengua extranjera en sus escuelas y, lo que es más preocupante, no parece haber consciencia de que este hecho 
afecta en su rendimiento escolar, su autoestima, su inmersión en la propia escuela y su posterior inmersión en nuestro país como ciudadanos chilenos. Con lo anterior, nuestro país se pone obstáculos para seguir un camino que hoy en día es tan inevitable como enriquecedor; convertirse en una sociedad multicultural.

\section{FINANCIAMIENTO}

Este trabajo forma parte del proyecto Fondecyt Regular $n^{\circ} 1190254$.

\section{REFERENCIAS}

Agencia de la calidad de la educación. Pisa 2018. Entrega de resultados Chile. Informe de resultados. 2019. <http://archivos.agenciaeducacion.cl/PISA_2018Entrega_de_Resultados_Chile.pdf.>.10 sep. 2020.

Bachman, Lyle y Adrian Palmer. Language Testing in Practice. Oxford: Oxford University Press, 1996. Impreso.

Bachman, Lyle. Fundamental Considerations in Language Testing. Oxford: Oxford University Press, 1990. Impreso.

Bereiter, Carl y Marlene Scardamalia. The psychology of written composition. New York: Routledge, 2009. Impreso.

Bizama, Marcela, y otros. "Conciencia sintáctica y comprensión de lectura en niñez vulnerable". Revista Latinoamericana de Ciencias Sociales, Niñez y Juventud (2017): 219-232. Impreso.

Blackedge, Adrian y Angela Creese. "Translanguaging in mobility". Canagarajah, Suresh. The Routledge Handbook of Migration and Language. London and New York: Routledge, 2017.

Byram, Michael. From Foreign Language education to education for intercultural citizenship. Clevedon: Multilingual Matters, 2007. Impreso.

Canagarajah, Suresh. "Lingua Franca English, multilingual communities, and language acquisition". Modern Language Journal (2007): 923-939. Impreso.

Canagarajah, Suresh. "Translingual writing and teacher development in composition". College English (2016): 265-273. Impreso.

Canagarajah, Suresh. The Routledge Handbook of Migration and Language. London and New York: Routledge, 2017. Impreso.

Canagarajah, Suresh. Translingual Practice: Global Englishes and Cosmopolitan Relations. London: Routledge, 2013. Impreso.

Canale, Michael. "From communicative competence to communicative language pedagogy". Richards, Jack y R. W. Schmidt. Language and Communication. Harlow: Longman, 1983. 2 -27. Impreso.

Cogo, Alessia y Martin Dewey. Analysis English as lingua franca: a corpus-driven investigation. New York: Continuum, 2012. Impreso. 
Artículo. Gloria Toledo Vega, Francisco Quilodrán, Miguel Olivares, Julio Silva. "Perspectivas actuales para el fomento del aula transcultural en Chile".

Collier, Catherine y John J. Hoover. "Sociocultural Considerations When Referring Minority Children for Learning Disabilities". Learning Disabilities Focus 3.1 (1987): 39-45. Impreso.

Collier, Virginia y Wayne Thomas. "Predicting Second Language Academic Success in English Using the Prism Model". Cummins, Jim y Chris Davison. International Handbook of English Language Teaching. Nueva York: Springer, 2007. 333-348. Impreso.

Connor, Ulla, Ed Nagelhout y William V. Rozycki. Contrastive Rhetoric: Reaching to Intercultural Rhetoric. Amsterdam: John Benjamins, 2008. Impreso.

Connor, Ulla. "Intercultural rhetoric research: beyond texts". Journal of English for Academic Purposes (2004): 291-304. Impreso.

Consejo de Europa. Marco común europeo de referencia para las lenguas: aprendizaje, enseñanza, evaluación. Madrid: Ministerio de Educación, Cultura y Deporte, 2002.

Cook, Vivian James . "Multi-competence". Robinson, Peter. The Routledge Encyclopedia of Second Language Acquisition. London and New York: Routledge, 2013. 447-450. Impreso.

Cook, Vivian James. "Premises of Multicompetence". Cook, Vivian James y Li Wei. The Cambridge Handbook of Linguistic Multicompetence. Cambridge: Cambridge University, 2016. 1-25. Impreso.

Cook, Vivian James. "The poverty of the stimulus argument and multi-competence". Second Language Research (1991): 103-117. Impreso.

Cook, Vivian James. Effects of the L2 on the L1. Clevedon: Multilingual Matters, 2003. Impreso.

Corson, D. "Language across the curriculum." Spolsky, Bernard. Concise encyclopedia of educational linguistics. Oxford: Pergamon, 1999. 323-325. Impreso.

Cots, Josep Maria, y otros. Plurilingüismo e interculturalidad en la escuela: reflexiones y propuestas didácticas. Barcelona: Horsori, 2010.

Cummins, Jim. "The influence of bilingualism on cognitive growth: a synthesis of research findings and explanatory hypotheses". Working Papers on Bilingualism (1976): 1-43. Impreso.

Cummins, Jim. Bilingualism and Special Education: Issues in assessment and pedagogy. San Diego: College Hill, 1984. Impreso.

Cummins, Jim. Language, Power and Pedagogy: Bilingual children in the crossfire. Clevendon: Multilingual Matters, 2000. Impreso.

Cummins, Jim. Negotiating Identities: Education for empowerment in diverse society. Los Angeles California: Association for Bilingual Education, 2001. Impreso.

Cummins, Jim. "De la alfabetización a la multialfabetización: el diseño de entornos de aprendizaje para la generación de conocimiento en la sociedad de la información". Sierra Plo, Juan Manuel y David Lasagabaster Herrarte. Multilingüismo y multiculturalismo en la escuela. Barcelona: ICE de la Universidad de Barcelona/ Horsori, 2005. 113-132. Impreso.

Cushing, Sara. Assessing Writing. Cambridge: Cambridge University Press, 2002. Impreso.

Dakin, Justine. "Writing bilingual stories: developing children's literacy through home languages". Mallows, David. Innovations in English Language Teaching for Migrants and refugees. London: British Council, 2012. 11-22. Impreso.

Douglas, Dan. Assessing language for specific purposes. Cambridge: Cambridge University Press, 2000. Impreso. 
Education First. Índice del EF English Proficiency. 2019. mayo de 2020. $<$ https://www.ef.com/cl/epi/>.

Grabowski, Joachim. "Writing and Speaking: Common grounds and differences toward a regulation theory of written language production". Levi, Michael y Sarah Ransdell. The science of writing. NJ: Lawrence Erlbaum Associates, 1996.

Hawkings, M. y A. Cannon. "Mobility, Language and Schooling". Canagarajah, Suresh. The Routledge Handbook of Migration and Language. New York: Routledge, 2017. 519-539. Impreso.

Hayes, John. "A new framework for understanding cognition and affect in writing". Levy, Michael y Sarah Ransdell. The Science of Writing. N.J.: Lawrence Erlbaum Associates, 1996.

Herdina, Philip y Ulrike Jessner. A Dynamic Model of Multilingualism: Perspectives of Change in Psycholinguistics. Clevedon: Multilingual Matters, 2002. Impreso.

Huot, Brian. "Toward a new theory of writing assessment". College Composition and Communication (1996): 549-566. Impreso.

Kaneva, Dimitrina. "Teaching and learning in diverse school context: the journeys of three newly-arrived students". Mallows, David. Innovations in English Language Teaching for Migrants and refugees. London: British Council, 2012. 49-64.

Kaplan, Robert B. "Cultural Thought Patterns in Inter-cultural Education". Language Learning 16 (1966): 1-20. Impreso.

Kubota, Ryoku y Al Lehner. "Toward critical contrastive rhetoric". Journal of Second Language Writing (2004): 7-27. Impreso.

Kubota, Ryoku y Al Lehner. "Response to Ulla Connor's Comments". Journal of Second Language Writing (2005): 137 - 143. Impreso.

Kubota, Ryoku. "Critical Approaches to Theory in Second Language Writing: A case of critical contrastive rhetoric." Silva, Tony y Paul Matsuda. Practicing theory in second language writing. West Lafayette, IN: Parlor Press, 2010. 191-208.

Kubota, Ryoku. "Cross-cultural perspectives on writing: Contrastive rhetoric." Hornberger, Nancy y Sandra McKay. Sociolinguistics and language education. Clevedon: Multilingual Matters, 2010. 265-289.

Lewkowicz, J. "Investigating authenticity in language testing." Tesis Doctoral. 1997.

Lorente, Beatriz. "Language-in-education policies and mobile citizens". Canagarajah, Suresh. The Routledge Handbook of Migration and Language. London and New York: Routledge, 2017. Impreso.

Lyster, Roy. Learning and teaching Languages Through Content. A counterbalanced approach. Amsterdam: John Benjamins, 2007. Impreso.

Maley, A. "ELF: A teacher's perspective". Language and Intercultural communication (2009): 187-200. Impreso.

Mallows, David. Innovations in English Language Teaching for Migrants and refugees. London: British Council, 2012. Impreso.

Mata, Francisco, José Gallego y Carlos Mieres. "Habilidades lingüísticas y comprensión lectora. Una investigación empírica”. Bordón (2007): 153-166. Impreso.

Mehmedbegović, Dina. "In search of high-level learner engagement: autobiographical, approaches with children and adults". Mallows, David. Innovations in English Language Teaching for Migrants and Refugees. London: British Council, 2012. 6578. 
Artículo. Gloria Toledo Vega, Francisco Quilodrán, Miguel Olivares, Julio Silva. "Perspectivas actuales para el fomento del aula transcultural en Chile".

MINEDUC. Curriculum Nacional. 2019. 20 de abril de 2020. $<$ https://curriculumnacional.mineduc.cl/614/w3-article-178042.html $>$.

National Writing Project y Carl Nagin. Because writing matters. Improving student writing in our schools. San Francisco: Jossey-Bass, 2006.

Ochoa, Salvador Hector, Bernadette D. Rivera y M. Paige Powell. "Factors Used to Comply with the Exclusionary Clause with Bilingual and Limited-English-Proficient Pupils: Initial Guidelines". Learning Disabilities Research and Practice 12.3 (1997): 161-167. Impreso.

Pavlenko, Aneta. "“We have room for but one language here": Language and national identity in the US as the turn of the 20th century." Multilingua (2002): 163-196.

Pearson, Christine. Controversies in second Language Writing. Dilemmas and Decisions in Research and Instruction. Muchigan: Multilingual Writers, 2013.

Prior, Paul. "A sociocultural theory of writing." MacArthur, C., S. Graham y J. Fitzgerald. Handbook of writing research. New York: The Guilford Press, 2006.

Rhodes, Robert, Salvador Ochoa y Samuel Ortiz. Assessing Culturally and Linguistically Diverse Students: A Practical Guide. New York: Guildford Press, 2005.

Robinson, Peter. The Routledge encyclopedia of second language acquisition. New York: Routledge, 2013. Impreso.

Roessler, Pablo, y otros. "Acceso e Inclusión de Personas Migrantes en el Ámbito Educativo (N.o 2).” 20 de Enero de 2020. Migración en Chile. 20 de Enero de 2020. $<$ https://www.migracionenchile.cl/wp-content/uploads/2020/06/Informe-2Educación-2020.pdf $>$.

Roseberry-McKibbin, Celeste. Multicultural Student with special language needs. Oceanside CA: Academic Communication Associates, 2002. Impreso.

Scott, Virginia. "Multi-competence and language teaching." Cook, Vivian James y Li Wei. The Cambridge Handbook of Linguistic Multi-competence. Cambridge: Cambridge University Press, 2016. 445-460.

Selinker, Larry. "Interlanguage". Product Information International Review of Applied Linguistics in Language Teaching (1972): 209-241. Impreso.

Shepherd, Sam. "Responsive teaching and learner centredness". Mallows, David. Innovations in English Language Teaching for Migrants and refugees. London: British Council, 2012. 165-176. Impreso.

Slomp, David. "Challenges in assessing the development of writing ability theories, constructs and methods". Assessing Writing (2012): 81-91. Impreso.

Smith, William, et al. "Some effects of varying the structure of the topic on college students' writing." Written Communication (1985): 73-89.

Urciuoli , B. y C. LaDousa. "Language management /labour." Annual Review of Anthropology (2013): 175-190. Impreso.

Zhu, Hua. "Interculturality: Reconceptualizing cultural membership and identities." Dervin, F. y K. Risager. Researching Identity and Interculturality: Towards a More Reflexive and Critical Methodology. London: Routledge, 2015. 109-124.

$\mathrm{Zhu}$, Hua. Exploring Intercultural Communication: Language in action. London: Routledge, 2014. Impreso. 